\title{
Supplementa zu den Jüdischen Schriften aus hellenistisch-römischer Zeit
}

\author{
Herausgegeben von \\ Hermann Lichtenberger und Gerbern S. Oegema
}

Mit den "Supplementa zu den Jüdischen Schriften aus hellenistisch-römischer Zeit « (Band VI.I-3) geht ein bereits im Jahre 1974 vom Gründer der Reihe "Jüdische Schriften aus hellenistisch-römischer Zeit«, Prof. Dr. Werner Georg Kümmel (gest. I995), formulierter Wunsch in Erfüllung, die Reihe beim Abschluß mit einem Einführungs-, Bibliographie- und Registerband zu erschließen.

Die Ergänzungsbände wurden in den Jahren 1996-1999 mit Forschungsmitteln der Deutschen Forschungsgemeinschaft am Institut für antikes Judentum und hellenistische Religionsgeschichte der Eberhard-Karls-Universität Tübingen (Prof. Dr. H. Lichtenberger/PD Dr. Drs. G.S. Oegema) in Zusammenarbeit mit dem Fachbereich Theologie der Johannes-Gutenberg-Universität Mainz (Prof. Dr. F. W. Horn) und der Rheinischen Friedrich-Wilhelms-Universität Bonn (Dr. H. Löhr) erstellt.

Das Projekt hat zum Ziel, eine Einführung, eine Bibliographie und ein Register zu erstellen, um die Apokryphen und Pseudepigraphen für die alt- und neutestamentliche Wissenschaft, die Orientalistik und die Judaistik zu erschließen, und wird in den Jahren I999-200I als Band VI.I-3 in der Reihe JSHRZ erscheinen.

Die geplante Einfübrung der JSHRZ, bearbeitet von Hermann Lichtenberger, Ulrike Mittmann-Richert und Gerbern S. Oegema, stellt eine Einführung in das jüdische außerqumranische und nichtrabbinische Schrifttum dar (außer Philo, Josephus, etc.). Sie bietet eine literaturgeschichtliche Einleitung und eine Geschichte der Editionen und Kommentare der Apokryphen und Pseudepigraphen. Sie ordnet literarisch und historisch die Texte und bespricht ihre Bedeutung für das antike Judentum und das frühe Christentum. Sie entfaltet die theologischen Hauptthemen (z. B. Einführung, Gott und Mensch, Angelologie und Dämonologie, Eschatologie und Soteriologie, Thora und Ethik, Politik und Gesellschaft).

Die Bibliographie der JSHRZ, bearbeitet von Andreas Lehnardt, ist aktualisiert bis 1997 und enthält nun auch hebräisch- und slawischsprachige Sekundärliteratur, erschließt sie alphabetisch und systematisch und bietet einen Zugang zum neuesten Stand der Forschung.

Das geplante Register der JSHRZ, bearbeitet von Friedrich Wilhelm Horn, Hermut Löhr, Heike Omerzu und Claudia Büllesbach, wird folgende Unterrubriken enthalten: Sachen, Namen, Stellen. Das Sachregister wird ermöglichen, in die religiösen Ideen, Vorstellungen und Motive der einzelnen Schriften einzuführen. Daneben sollen hier auch geographische Bezeichnungen aufgenommen werden. Das $\mathrm{Na}$ menregister soll die in den Schriften aufgeführten biblischen oder außerbiblischen Gestalten bzw. Personen erfassen. In den Anmerkungen der einzelnen Bände finden sich Verweise auf Parallelstellen oder vergleichbare Aussagen in der biblischen und außerbiblisch-jüdischen bzw. -christlichen wie auch in der pagan-antiken Literatur. Der dort erarbeitete Schatz an religions- und traditionsgeschichtlichem Material soll auch in das Register aufgenommen werden.

Tübingen, im Oktober 1998

Hermann Lichtenberger Gerbern S. Oegema 